\title{
MULTIOBJECTIVE RELIABILITY ALLOCATION IN MULTI-STATE SYSTEMS: DECISION MAKING BY VISUALIZATION AND ANALYSIS OF PARETO FRONTS AND SETS
}

\author{
E. $\mathrm{Zio}^{1}{ }^{2},{ }^{2}$, R. Bazzo ${ }^{2}$ \\ ${ }^{1}$ Ecole Centrale Paris- Supelec, Paris, France \\ Chair "Systems Science and Energetic Challenge" \\ European Foundation for New Energy - EDF \\ enrico.zio@ecp.fr \\ ${ }^{2}$ Politecnico di Milano, Milano, Italy \\ Dipartimento di Energia \\ enrico.zio@polimi.it
}

\begin{abstract}
Reliability-based design, operation and maintenance of multi-state systems lead to multiobjective (multicriteria) optimization problems whose solutions are represented in terms of Pareto Fronts and Sets. Among these solutions, the decision maker must choose the ones which best satisfy his preferences on the objectives of the problem. Visualization and analysis of the Pareto Fronts and Sets can help decision makers in this task. In this view, a recently introduced graphical representation, called Level Diagrams, is here used in support of the analysis of Pareto Fronts and Sets aimed at reducing the number of non-dominated solutions to be considered by the decision maker. Each objective and design parameter is represented on separate "synchronized" diagrams which position the Pareto front points according to their proximity to ideal preference points and on the basis of this representation a two-step front reduction procedure is proposed. An application to a redundancy allocation problem of literature concerning a multi-state system is used to illustrate the analysis.
\end{abstract}

Keywords: Multi-State System Redundancy Allocation, Reliability, Availability, Multiobjective Decision Making, Pareto Front, Pareto Set, Level Diagrams

This work has been funded by the Foundation pour une Culture de Securite Industrielle of Toulouse, France, A0-2009-04. 


\subsection{INTRODUCTION}

Solutions to multiobjective problems in reliability-based multi-state system design, operation and maintenance are not unique as they have to satisfy simultaneously various conflicting objectives. The set of solutions for consideration of the decision maker (DM) forms the Pareto Set $^{1}$ in the space of the decision variables, and the Pareto Front ${ }^{2}$ in the space of the objectives.

In practice, the solution to a multiobjective optimization problem is found by a search algorithm as a discrete approximation of the Pareto Front, from which the DM has to select one, or more, solutions of preference. For this task to be successful, the DM should be confronted with a relatively small number of solutions representative of feasible alternatives on the Pareto Front.

In this view, visualization techniques can represent valuable tools for analyzing the multidimensional Pareto Front and Set. For two-dimensional (and at times for three-dimensional) problems, it is usually possible to make an accurate graphical analysis of the Pareto Front and Set, but this becomes soon impractical for higher dimensions. Some of the most common techniques proposed for multidimensional visualization are [1]:

- Scatter diagrams: The visualization consists of an array of scatter diagrams arranged in the form of an $s \mathrm{X} s$ matrix, where $s$ is the number of objectives. Each objective function defines one row and one column of the matrix. The complexity of the representation increases notably with the number of dimensions.

- Parallel coordinates: A multidimensional point is plotted in a two-dimensional graph, one for the objective functions and one for the decision variables. Each dimension of the original data is transformed into a coordinate in the two-dimensional plot. This is a very compact way of presenting multidimensional information, but it soon loses clarity with large sets of data and the analysis becomes difficult.

- Interactive Decision Maps [7]: Two-dimensional projections of the objective function space are used to display decision maps of contour lines parametrized by a third, colour-associated objective; the process of parametrizing the two-dimensional contours can become timeconsuming and cumbersome when many objectives are involved.

\footnotetext{
${ }^{1}$ The so-called Pareto Set is the set containing all non-dominated solutions resulting from multiobjective optimization problems. Multiobjective optimization problems generally do not have a unique solution, but rather a set of solutions, which dominate all others but among them none is better. Dominance and non-dominance is determined by pair-wise vector comparisons of the multiobjective values corresponding to the pair of solutions under comparison.

${ }^{2}$ The Pareto Front is the set of the values of the objective functions in correspondence of the solutions of the Pareto Set.
} 
Recently, Level Diagrams ${ }^{3}$ have been introduced for visualizing multidimensional Pareto Fronts and Sets [2]; they can be employed a priori, interactively or a posteriori of the optimization process to help the DM defining his or her preferences during the solution selection phase. The visualization is based on a metric distance from an ideal solution which optimizes all objectives simultaneously; a solution colouring procedure can be adopted to visualize the DM preferences.

In the present work, Level Diagrams are drawn for the Pareto Front and Set of a multi-state system redundancy allocation problem of literature which involves three conflicting objectives: system availability to be maximized, system cost and weight to be minimized [8]. The decision variables define the system configurations, each variable indicating the number of components of a particular type allocated in the configuration to provide redundancy.

The Level Diagrams analysis is then exploited for establishing a two-step procedure of reduction of the number of possible solutions represented, which renders it easier for the DM to apply his or her preferences.

The remainder of the paper is organized as follows. Section 16.2 recalls the main ideas behind the Level Diagrams representation and the related procedure [2]. In Section 16.3, the multi-state system redundancy allocation problem of interest is formulated [8]. Section 16.4 reports the analysis of the Pareto Front and Set using Level Diagrams, with the proposed procedure of front reduction. Finally, some conclusions are drawn in Section 16.5.

\footnotetext{
${ }^{3}$ Level Diagrams are a recently introduced technique for visualizing Pareto Front and Sets, which will be further explained in the chapter.
} 


\subsection{LEVEL DIAGRAMS REPRESENTATION OF PARETO FRONTS AND SETS}

\subsubsection{Pareto Front and Set}

Without loss of generality, let us consider a multiobjective minimization problem $\min _{\theta \in D} J(\theta)$

where $\theta=\left[\theta_{1}, \ldots ., \theta_{p}\right] \in D$ is the vector of decision variables, $D$ is the decision space and $J(\theta)=\left[J_{1}(\theta), \ldots, J_{s}(\theta)\right]$ is the vector of objective functions.

Generally, there is a set of solutions to (1) dominating all others but among them none is superior. Dominance is determined by comparing the values of the multiple objectives in correspondence of pairs of solutions: solution $\theta^{1}$ dominates solution $\theta^{2}$, if

$\forall i \in\{1, \ldots, s\}, J_{i}\left(\theta^{1}\right) \leq J_{i}\left(\theta^{2}\right) \wedge \exists k \in\{1, \ldots ., s\}: J_{k}\left(\theta^{1}\right)<J_{k}\left(\theta^{2}\right)$.

The set $\Theta_{p}$ of non-dominated solutions is called the Pareto Set; the vector $J(\theta)$ of the values of the objective functions in correspondence of the solutions $\theta \in \Theta_{p}$, defines the Pareto Front.

\subsubsection{Level Diagrams}

From the discrete set of Pareto solutions obtained by a multiobjective optimization algorithm, the decision maker is called to choose the best solution according to his or her preferences. This task can be quite difficult for high dimensional problems and must be aided by effectively visualizing the results of the multiobjective optimization.

Level Diagrams represent an interesting visualization tool which allows classifying the Pareto solutions according to their distance from the ideal solution, i.e., the one which optimizes all objectives simultaneously [2].

Assuming, in general, that $l$ objectives are to be minimized and $m$ maximized, with $s=l+m$, each objective is normalized with respect to its minimum or maximum values on the Pareto Front:

$\overline{J_{i}}(\theta)=\frac{J_{i}(\theta)-J_{i}{ }^{\min }}{J_{i}^{\max }-J_{i}{ }^{\min }} \quad i=1, \ldots . ., l$

and

$\overline{J_{i}}(\theta)=\frac{J_{i}^{\max }-J_{i}(\theta)}{J_{i}^{\max }-J_{i}^{\min }} \quad i=1, \ldots, m$

so that now, 
$0 \leq \overline{J_{i}}(\theta) \leq 1 \quad i=1, \ldots ., s$.

The value $\overline{J_{i}}(\theta)=0$ means that the solution $\theta$ gives the best value for the $i-t h$ objective whereas the value $\overline{J_{i}}(\theta)=1$ corresponds to the solution $\theta$ giving the worst value for the $i-t h$ objective. The distance to the ideal point $\theta^{*}: \overline{J_{i}}\left(\theta^{*}\right)=0 \forall i=1, \ldots ., s$

can be computed with different norms, giving different views on the characteristics of the Pareto Front [2]. The norm here considered is the 1-norm :

$\|\bar{J}(\theta)\|_{1}=\sum_{i=1}^{s}\left|\overline{J_{i}}(\theta)\right|$, with $0 \leq\|\bar{J}(\theta)\|_{1} \leq s$

The Level Diagrams are drawn as follows: each objective $\left(J_{i}\right)$ and each decision variable $\left(\theta_{j}\right)$ is plotted separately; the $\mathrm{X}$ axis corresponds to the objective or the decision variable in physical units of measurement, while the $\mathrm{Y}$ axis corresponds to the value $\|\bar{J}(\theta)\|_{1}$ for all the graphs; this means that all the plots are synchronized with respect to the $\mathrm{Y}$ axis, i.e., all the information for a single point of the Pareto Set will be plotted at the same level of the Y axis. 


\subsection{THE MULTI-STATE SYSTEM REDUNDANCY ALLOCATION PROBLEM}

The redundancy allocation problem (RAP) here considered regards the selection of the system redundancy configuration that maximizes availability and minimizes cost and weight. The system is decomposed in a number of subsystems, for each of which there are multiple component choices to be allocated in redundancy [4], [2].

The RAP case study here considered is taken from [8]. The system is made of $u=5$ units (subsystems) connected in series; redundancy can be provided to each unit by selecting components from $m_{i}$ types available in the market, $i=1, \ldots ., 5$. Each component can be in two states: functioning at nominal capacity or failed, at zero capacity. The collective performance of these binary components leads to a multi-state system behaviour. The types of components available are characterized by their availability, nominal capacity, cost and weight in arbitrary units (Table 16.1). Without loss of generality, component capacities are measured in terms of percentage of the maximum system demand. The different demand levels for a given period are given in Table 16.2.

\begin{tabular}{|c|c|c|c|c|c|}
\hline $\begin{array}{c}\text { Subsystem } \\
i\end{array}$ & $\begin{array}{c}\text { Component } \\
\text { Type } j\end{array}$ & Availability & Capacity (\%) & Cost & Weight \\
\hline \multirow{7}{*}{1} & 1 & 0.980 & 120 & 0.590 & 35.4 \\
\hline & 2 & 0.977 & 100 & 0.535 & 34.9 \\
\hline & 3 & 0.982 & 85 & 0.470 & 34.1 \\
\hline & 4 & 0.978 & 85 & 0.420 & 33.9 \\
\hline & 5 & 0.983 & 48 & 0.400 & 34.2 \\
\hline & 6 & 0.920 & 31 & 0.180 & 34.3 \\
\hline & 7 & 0.984 & 26 & 0.220 & 32.6 \\
\hline \multirow{5}{*}{2} & 1 & 0.995 & 100 & 0.205 & 26.5 \\
\hline & 2 & 0.996 & 92 & 0.189 & 22.4 \\
\hline & 3 & 0.997 & 53 & 0.091 & 20.3 \\
\hline & 4 & 0.997 & 28 & 0.056 & 21.7 \\
\hline & 5 & 0.998 & 21 & 0.042 & 25.2 \\
\hline \multirow{3}{*}{3} & 1 & 0.971 & 100 & 7.525 & 42.1 \\
\hline & 2 & 0.973 & 60 & 4.720 & 41.7 \\
\hline & 3 & 0.971 & 40 & 3.590 & 40.8 \\
\hline
\end{tabular}




\begin{tabular}{|c|c|c|c|c|c|}
\hline & 4 & 0.976 & 20 & 2.420 & 39.6 \\
\hline \multirow{9}{*}{4} & 1 & 0.977 & 115 & 0.180 & 25.4 \\
\hline & 2 & 0.978 & 100 & 0.160 & 23.9 \\
\hline & 3 & 0.978 & 91 & 0.150 & 24.7 \\
\hline & 4 & 0.983 & 72 & 0.121 & 24.6 \\
\hline & 5 & 0.981 & 72 & 0.102 & 23.6 \\
\hline & 6 & 0.971 & 72 & 0.096 & 26.2 \\
\hline & 7 & 0.983 & 55 & 0.071 & 25.5 \\
\hline & 8 & 0.983 & 25 & 0.049 & 22.6 \\
\hline & 9 & 0.977 & 25 & 0.044 & 24.8 \\
\hline \multirow{4}{*}{5} & 1 & 0.984 & 128 & 0.986 & 15.4 \\
\hline & 2 & 0.983 & 100 & 0.825 & 15.3 \\
\hline & 3 & 0.987 & 60 & 0.490 & 14.9 \\
\hline & 4 & 0.981 & 51 & 0.475 & 15.0 \\
\hline
\end{tabular}

Table 16.1 : Characteristics of the components available on the market

\begin{tabular}{|l|l|l|l|l|}
\hline Demand (\%) & 100 & 80 & 50 & 20 \\
\hline Duration (h) & 4203 & 788 & 1228 & 2536 \\
\hline Duration (\%) & 0.48 & 0.09 & 0.14 & 0.29 \\
\hline
\end{tabular}

Table 16.2 : Demand levels

The three objectives in mathematical terms are:

Availability: $\quad \max _{\theta} J_{1}(\theta)=\max _{\theta}\left[\prod_{i=1}^{u} A_{i}\left(\theta_{i}\right)\right]$,

Cost : $\quad \min _{\theta} J_{2}(\theta)=\min _{\theta}\left[\sum_{i=1}^{u} \sum_{j=1}^{m_{i}} c_{i j} \theta_{i j}\right]$

and

Weight : $\quad \min _{\theta} J_{3}(\theta)=\min _{\theta}\left[\sum_{i=1}^{u} \sum_{j=1}^{m_{i}} w_{i j} \theta_{i j}\right]$

subject to: $1 \leq \sum_{j=1}^{m_{i}} \theta_{i j} \leq n_{\max , i}, \forall i=1, \ldots ., u$ 
where $A_{i}=$ availability of the $i-t h$ subsystem, $\theta=$ vector of the indexes of the system configuration, $\theta_{i j}=$ component of vector $\theta$, i.e., index of the $i-t$ component in $j-t h$ subsystem, $u=$ number of subsystems, $m_{i}=$ number of components in the $i-$ th subsystem, $n_{\max , i}=$ userdefined maximum number of redundant components that can be placed in subsystem $i, n_{i j}=$ number of components of type $j$ used in subsystem $i, c_{i j}, w_{i j}=$ cost and weight of the $j-t h$ type of component in subsystem $i$.

The problem has been solved in [10] using the MOMS-GA algorithm coupled with the Universal Moment Generating Function (UMGF) approach ([11], [6] and [5]) for computing the system availability (8). The resulting Pareto Front of 118 points is showed in Figure 16.1 in the objective functions space.

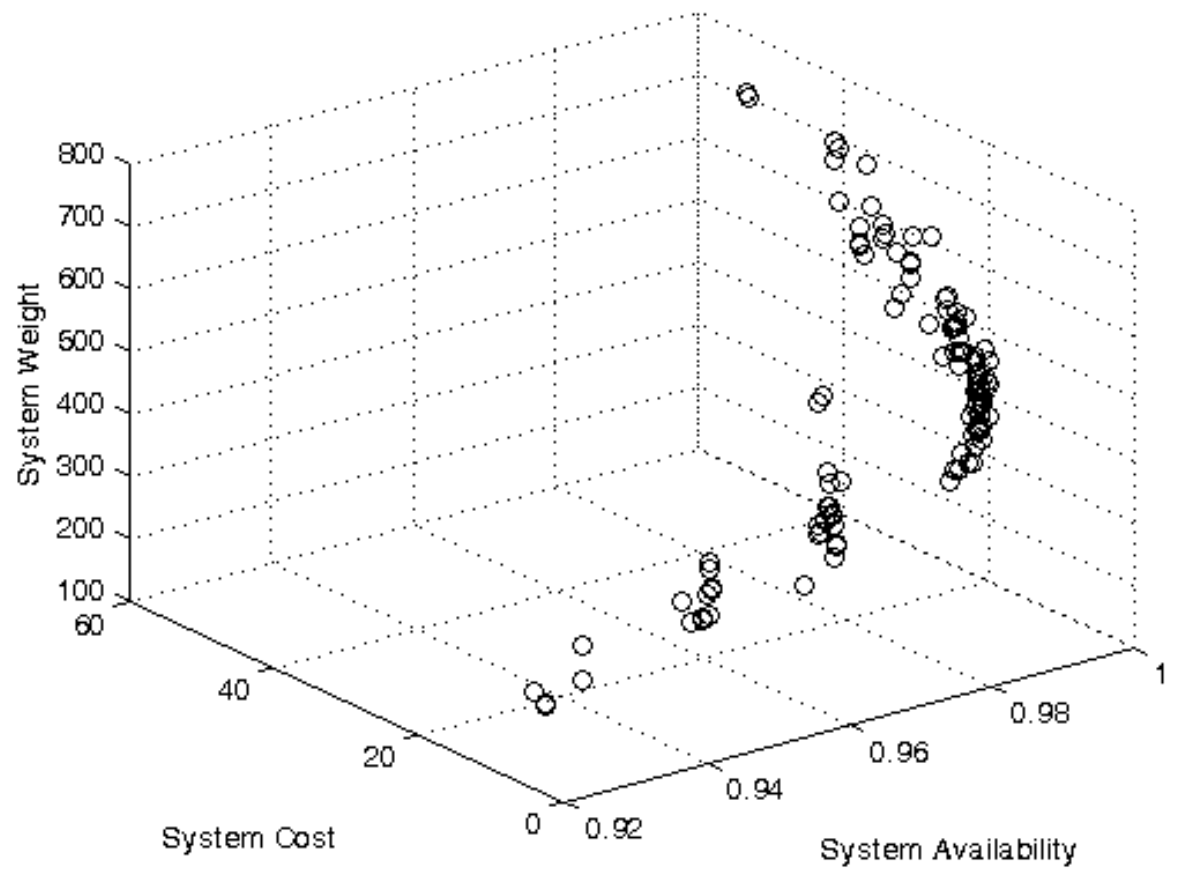

Figure 16.1: Pareto Front in the objective functions space, obtained by the MOMS-GA algorithm 


\subsection{VISUALIZATION WITH THE LEVEL DIAGRAMS}

\subsubsection{Objective Functions}

The Level Diagrams of the Pareto Front of Figure 16.1 are showed in Figure 16.2 for the system availability, cost and weight.
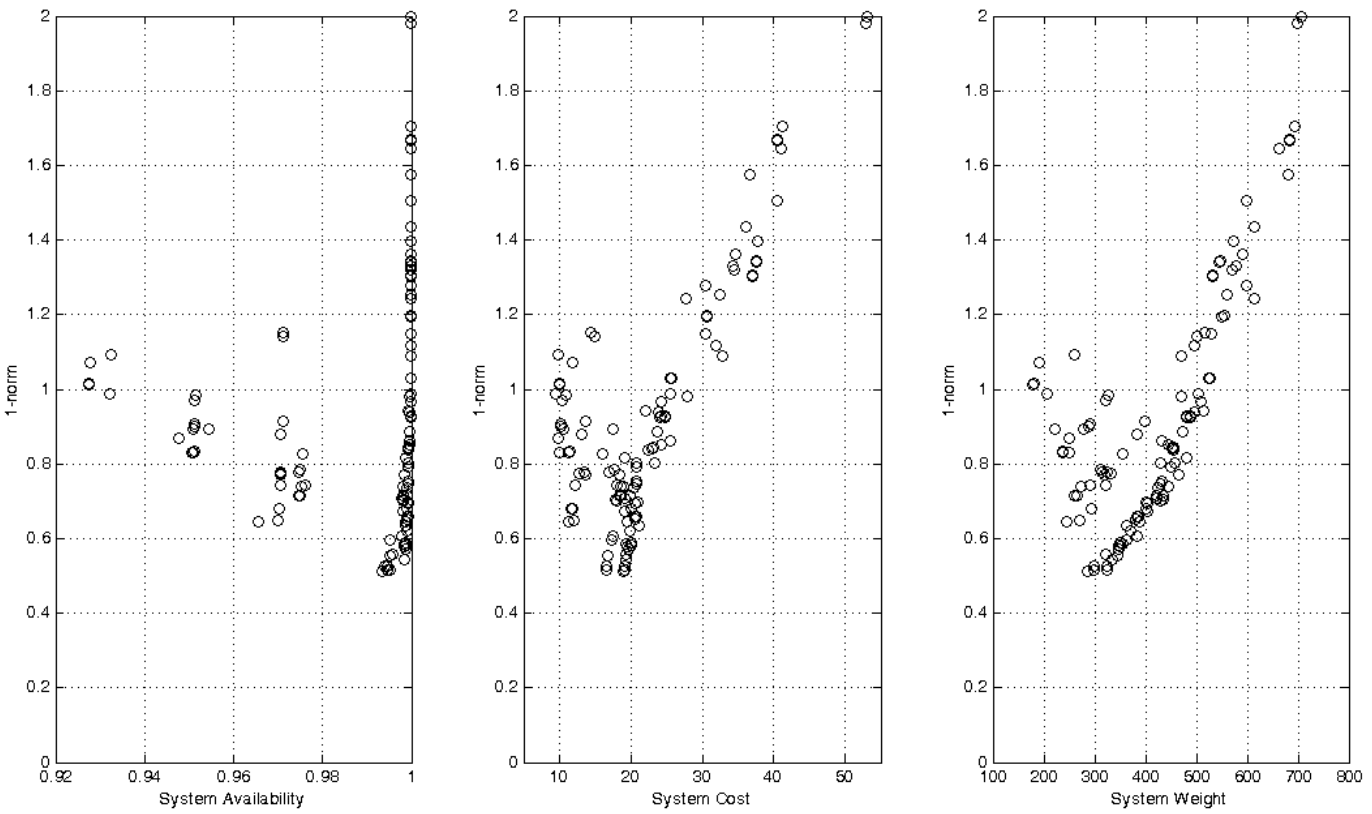

Figure 16.2: Level Diagram representation for availability objective, J1 (a-left), cost objective, J2 (b-center), weight objective, J3 (c-right)

In the Level Diagrams of all three objectives, one observes an initial decreasing trend of the norm value up to a minimum and then an increasing trend; this is reasonable, due to the conflict among the objectives. To understand this behaviour, let us consider for example Figure 16.2-a related to the availability objective: system design configurations of low availability (far from the optimal value) are characterized by small costs and weights (close to the optimal values) and as a result, the values of the 1-norm (7) are large; for values of the system availability increasing towards the optimal value, the norms decrease up to the minimum value, optimal with respect to all three objectives, after which the increase of cost and weight is such that the norms increase as the point in the Pareto Front moves away from the ideal one, optimal with respect to all three objectives.

The Level Diagrams representation allows also a qualitative evaluation of the sensitivity of the results. Looking again at Figure 16.2 for the system availability objective, one can observe that the 
absolute value of the slope of the ascending part is larger than in Figure 16.2-b and Figure 16.2-c for the cost and the weight objectives, respectively: for example, system availabilities above 0.99 are achieved by rapidly moving away from the ideal point of minimum norm, due to significant cost and weight increases.

An interesting feature is the presence of data clusters in the 1-norm Level Diagram of availability (Figure 16.2). This is due to the fact that whereas the cost and weight objectives grow "regularly" with increasing availability, the values that this latter objective can take are discontinuous, depending on the discrete redundant configurations which can be devised with different costs and weights but same availability values. This becomes clear if one considers Figure 16.3 in which the normalized values (3) or (4) of the three objectives (8), (9) and (10) are also plotted. Notice for example how at a given value of system availability (Figure 16.3-a) correspond very different values of weight, creating the vertical alignment that can be seen in the Level Diagrams of Figure 16.2 and Figure 16.3. Something similar occurs in Figure 16.3-b, where at cost values around 20 correspond various values of weight, with consequent vertical alignment of the 1-norm values. These vertical alignments are caused by solutions which have approximately the same value of the objective represented in the Level Diagram, but different values of the other objectives.
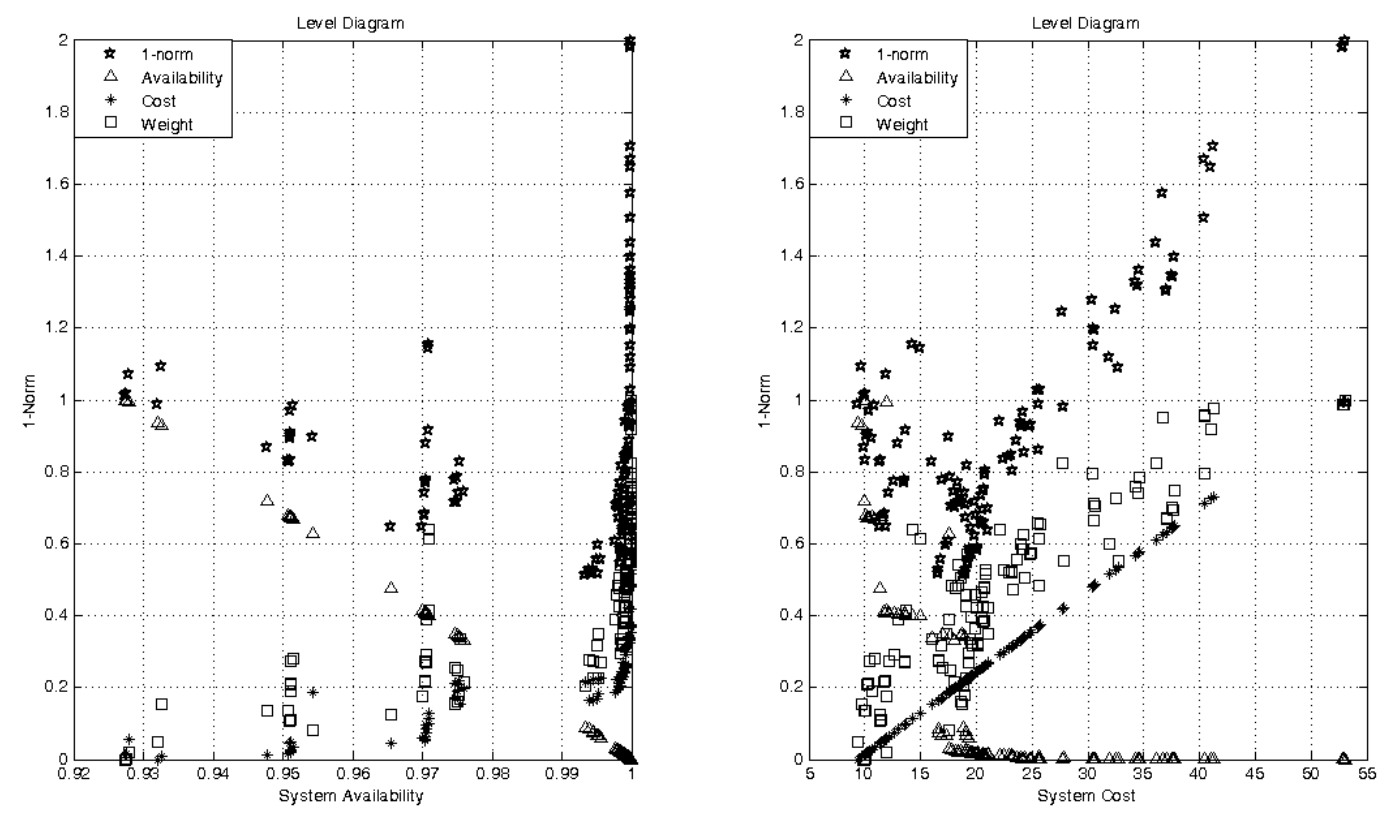

Figure 16.3 : Level Diagram for availability objective J1 and normalized objectives (a-left), cost objective J2 and normalized objectives (b-right)

The representation of the Pareto Front by means of Level Diagrams has identified the presence of system design configurations of similar values of availability but different cost and weight values. 
The observations thereby obtained suggest a Pareto Front reduction through the suppression of the configurations with higher values of 1-norm among those with similar values of one objective, availability. In Section 16.4.3, the results of this reduction will be shown to provide a less "crowded" Pareto Front, easier to be analysed by the DM for his or her preferences evaluation on the way to a final decision.

\subsubsection{Decision Variables}

In the case study considered, the decision variables define the system configurations in terms of the subsystem of redundant components. The configuration is contained in a vector of 29 discrete decision variables; each variable indicates the number of components of that particular type present in the configuration.

Figure 16.4 shows the Level Diagrams relative to the 7 decision variables characterizing the redundancy configuration of the first subsystem. Notice how some of the low capacity components (e.g., 6 and 7) are not selected in any of the Pareto Set configurations. On the contrary, the components of large capacity (the highest in the list of Table 16.1) are typically used, possibly in redundancy to ensure good values of availability. This is due to the definition of the multistate system availability, which considers not only the components availabilities but also their feeding capacities [6].
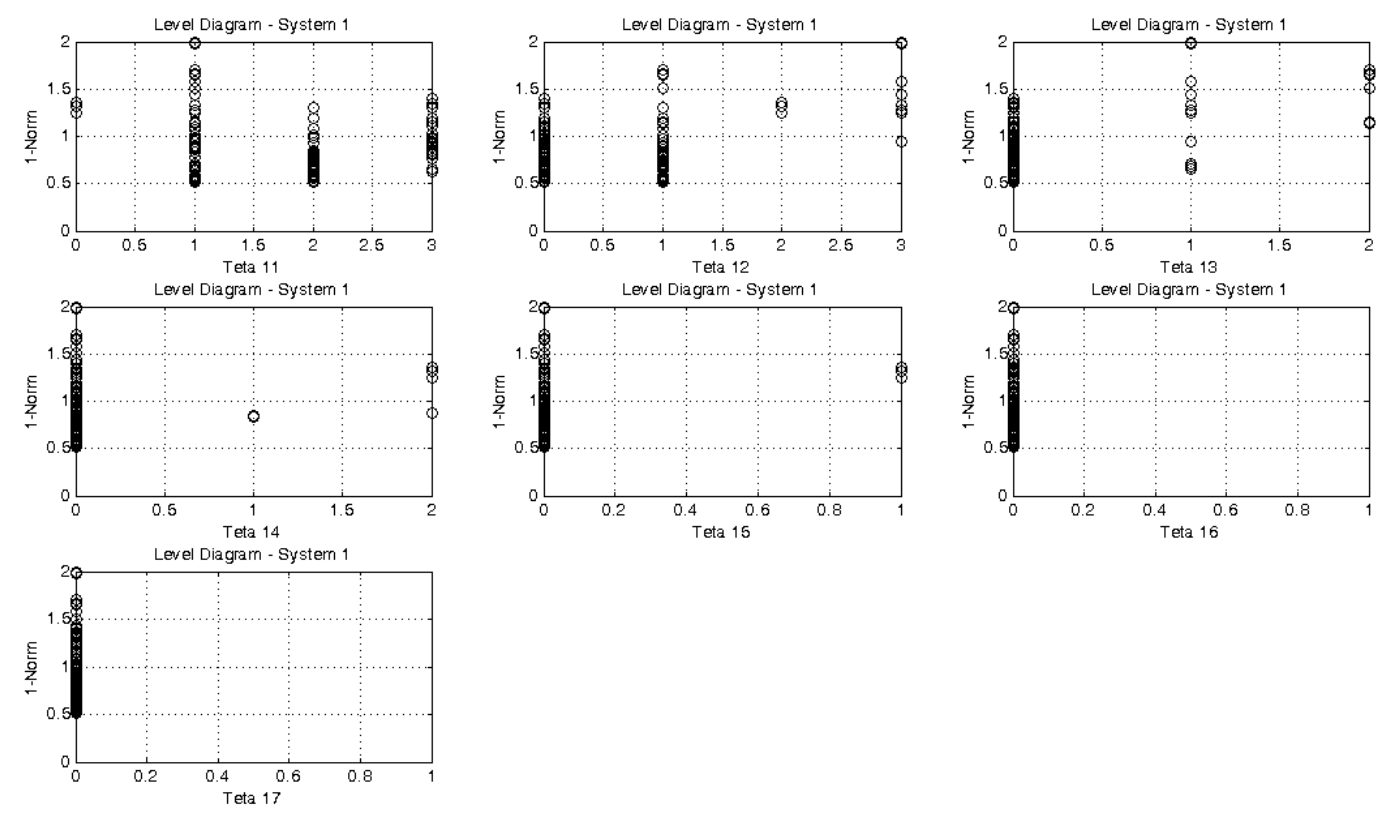

Figure 16.4 : Level Diagram representation of the 7 decisional variables values of subsystem 1 
In Figure 16.4, one also notices that to some decision variables values are associated a wide range of 1-norm values: for example, there is a large number of solutions in the Pareto Set with $\theta_{11}=1$ which are associated to different values of the 1-norm on the Level Diagrams, i.e., different distances from the optimal point, ideal with respect to all the three objectives (top-left graph). Solutions with $\theta_{11}=1$ are thus quite ubiquitous in the space of the objective functions (Figure 16.1) and the characteristics of the Pareto front may not be substantially affected if it were reduced to only the solutions with $\theta_{11}=1$.

\subsubsection{Pareto Front and Set Reduction}

The Pareto Front and Set generated by a search algorithm often result in a crowded set of dominant solutions gradually different among them; on the contrary, less crowded Pareto Front and Set would be beneficial to the DM who must analyze the different solutions and identify the preferred ones.

One way of proceeding to a reduction of the solutions contained in the Pareto Front and Set is that of focusing on the values of one objective function (in this case availability), and keep only few representative solutions of the clusters of solutions with approximately equal value of that objective function (availability) and different values of the other objective functions (cost and weight). The solutions retained as representative of a cluster at a given value of availability are those with lowest costs and weights. The clusters along the Pareto Front are then reduced to a few representative points different in terms of availability and optimal in terms of low costs and weights.

To systematically perform the Pareto Front reduction, a criterion must be established to define when two solutions on the Level Diagrams can be considered vertically aligned in a cluster of equal value of an objective (availability). To this purpose, let us consider a vector $J_{1}$ of length $n_{j}$ containing all the values of the availability objective of the Pareto Front sorted in ascending order, let $i_{\min }$ be the position in the vector $J_{1}$ of the optimal, ideal point (optimal with respect to all the three objectives), i.e., the solution with the lowest values of the 1-norm (7) and let norm 1 be the vector containing the values of the 1-norm (7) for each solution on the Pareto Front. Since the differences of the availability values of two successively ranked solutions, i.e., $J_{1}(i+1)-J_{1}(i)$ are very different in the two branches of the Front (Figure 16.2 and Figure 16.3), two distinct criteria are needed to decide whether two solutions can be considered vertically aligned in a cluster on the left and right of the minimum value of the norm. Then, in the vertical cluster alignment, solutions with a higher value of the 1-norm, i.e., with a worse overall system performance, are discarded. In 
this work, the solution $\theta(i+1)$ corresponding to $J_{1}(i+1)$ and to norm $1(i+1)$, belongs to a vertical cluster alignment if the previous solution $\theta(i)$ is such that:

$J_{1}(i+1)-J_{1}(i) \leq 10^{-3} \quad$ for $1 \leq i \leq i_{\min }$

i.e., on the right of the ideal point, while

$J_{1}(i+1)-J_{1}(i) \leq 10^{-5} \quad$ for $i_{\min }<i \leq n_{j}$

i.e., on the left of the ideal point. Then, the solution $\theta(i)$ is discarded if

norm $1(i+1)<\operatorname{norm} 1(i)$;

viceversa, the solution $\theta(i+1)$ is discarded if

norm $1(i+1)>$ norm $1(i)$.

The results of the reduction performed on the Pareto Front under analysis are plotted in Figure 16.5: the Pareto Front is reduced from the original 118 points to 52, with the clusters indeed reduced to individual best points, i.e., those of lowest 1-norm at basically equal availability values.

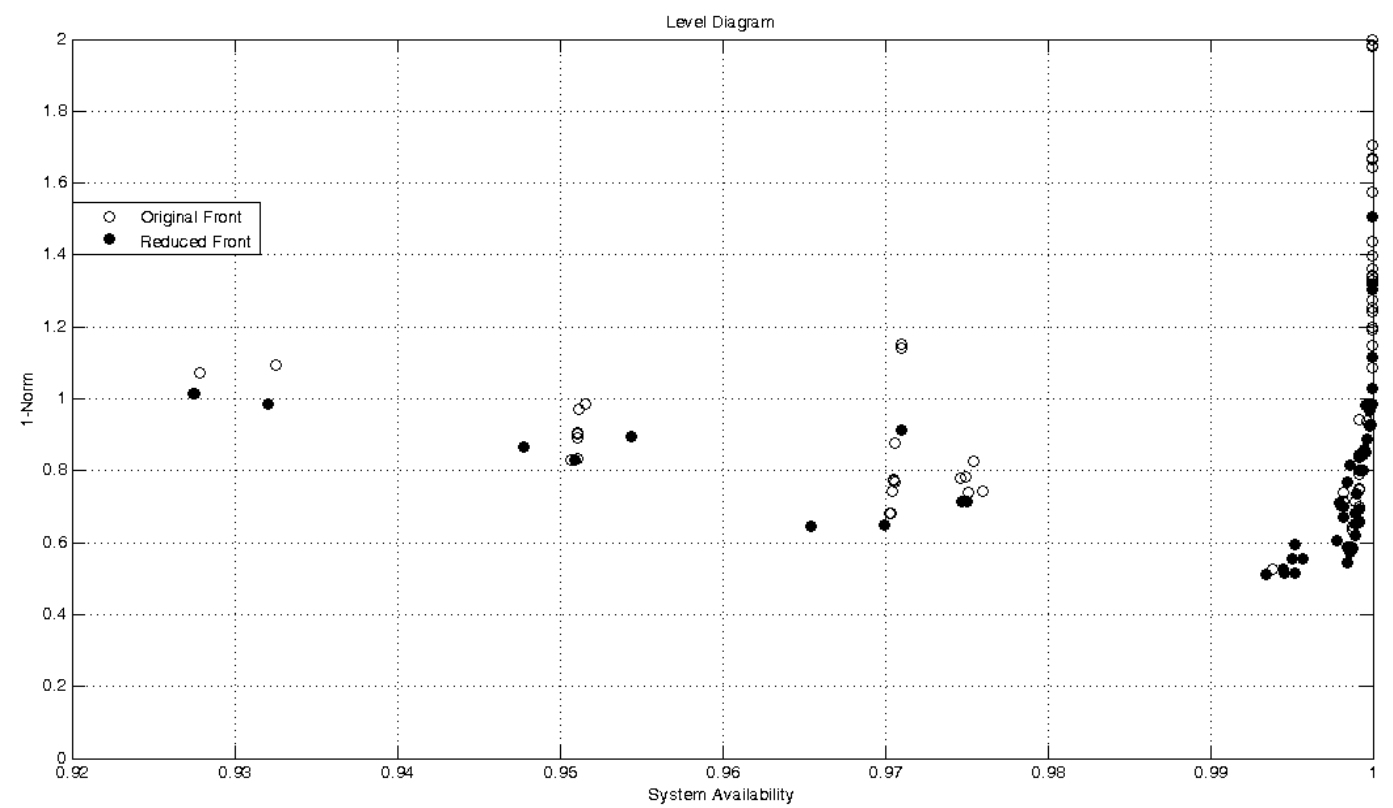

Figure 16.5 : 1-norm Level Diagram representation of the reduced Pareto Front for the availability objective, J1

In synthesis, the examination of the Pareto Front portrayed by Level Diagrams has allowed reduction by approximately $56 \%$ of the solutions, before imposing any preference or constraint.

A further reduction of the solutions contained in the Pareto Front and Set can be achieved by focusing on the values of selected decision variables. The solutions in the Pareto Front and Set can be reduced to those whose selected decision variables have given values, while maintaining the 
Front shape and characteristics. The underlying idea has been briefly introduced in Section 16.4.2, with reference to the decision variable $\theta_{11}$ at its value of 1 (top-left graph of Figure 16.4), but in general one may consider more than one decision variable.

The decision variables Level Diagrams can be used to guide the selection of the decision variables and their values to be subjected to reduction, because they visualize the different 1-norm values corresponding to the decision variable values, thus showing the extent to which the solutions characterized by a given value of a decision variable cover the variety of solutions on the Pareto Front. With reference to Figure 16.4 for subsystem 1, the decision variables and their values worth to be considered for guiding the reduction, because offering a wide coverage of different Pareto Front solutions, are: $\theta_{11}=1$ (top-left graph), $\theta_{12}=1$ (top-center graph), $\theta_{14}=0$ (middle-left graph), $\theta_{15}=0$ (middle-center graph); looking at the Level Diagrams related to the other subsystems (here not shown), also $\theta_{44}=0, \theta_{45}=0$ and $\theta_{54}=0$ would be worth consideration for driving the reduction. In the final selection of the decision variables and values for the reduction of the Pareto Front and Set solutions, particular attention must be given to preserving as much as possible the representation of the region closest to the minimum 1-norm value in the Level Diagrams because it contains the best solutions, of minimum distance from the ideal point. Applying these considerations to the original Pareto Front and Set reduces the solutions to only 36. Figure 16.6 shows, for example, the reduced 1-norm Level Diagram of the availability objective: notice, however, that although the shape is preserved, the clusters of solutions are not reduced to the best performing solutions, i.e., the minimum 1-norm solutions, as in the previous reduction procedure. 


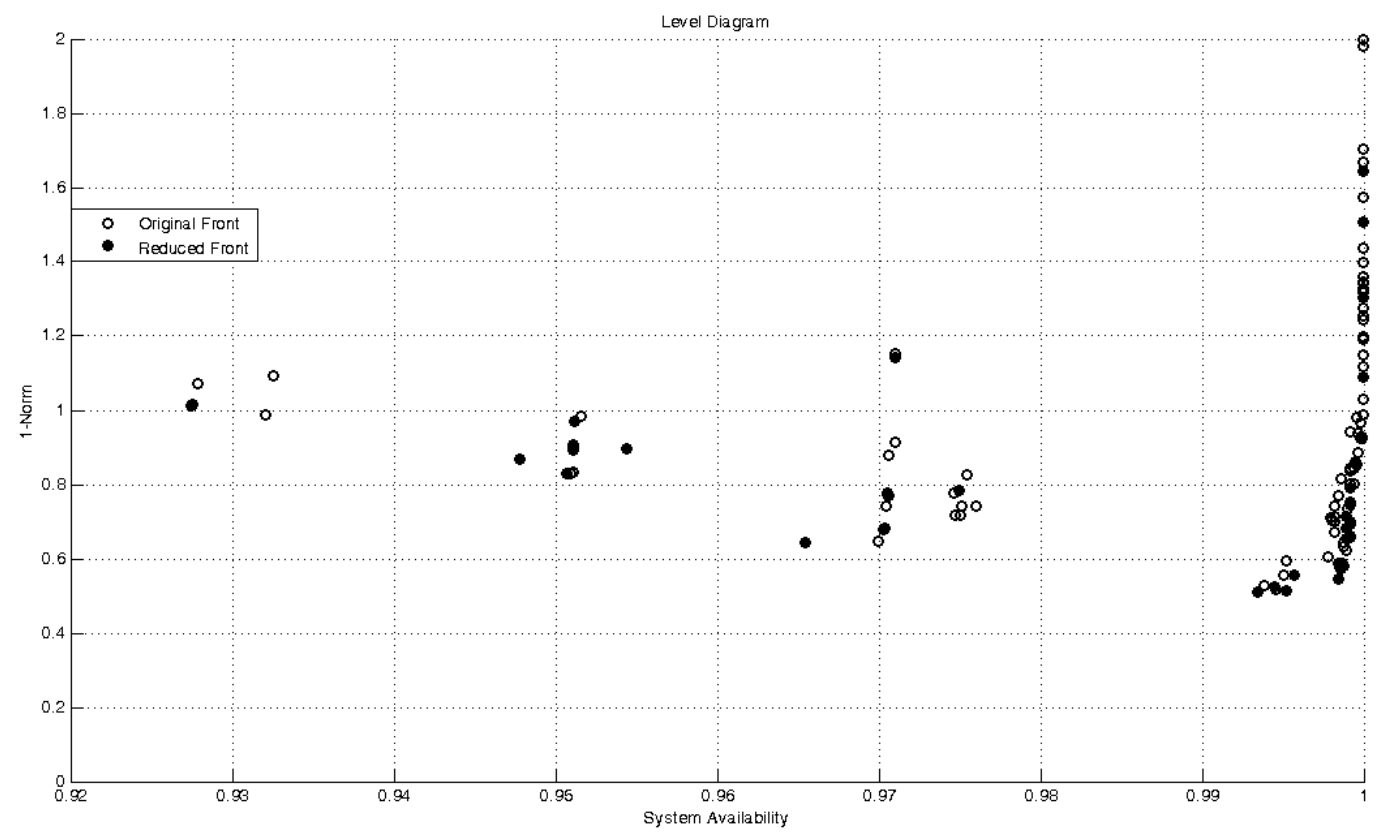

Figure 16.6: 1-norm Level Diagram representation of the reduced Pareto Front obtained by decision variables selection for the availability objective, J1

This suggests to apply the two reduction procedures in succession: the result is a Pareto Front and Set of only 19 solutions. Figure 16.7 shows the representation of the Pareto Front in terms of the Level Diagrams of the three objective functions.
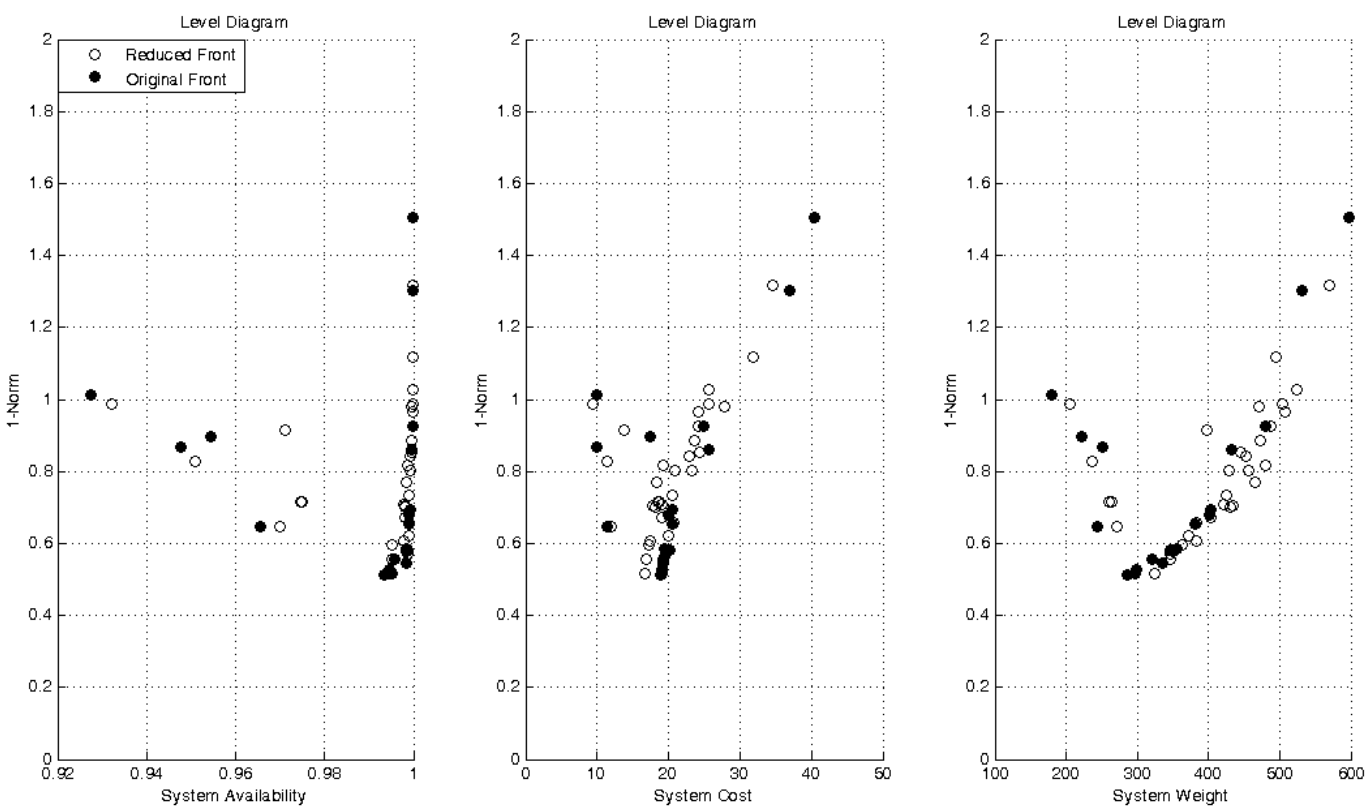

Figure 16.7 : 1-norm Level Diagram representation of the reduced Pareto Front obtained by the two-steps reduction, for the availability objective, J1 (a-left), the cost objective, J2 (b-center) and the weight objective, J3 (c-left) 
Looking for example at Figure 16.7-a, one can notice that the representation of the solutions in the region of the low 1-norm values is well preserved. Experiments performed by changing the selected decision variables and values have showed that the resulting reduced Pareto Front and Set are quite sensitive to these choices, in particular with respect to preserving the region of greatest interest, i.e., of low 1-norm values. 


\subsection{CONCLUSIONS}

Level Diagrams used in the analysis of the Pareto Front and Set of multiobjective optimization problems can drive their reduction by removal of less efficient solutions, while maintaining the Front characteristics. In particular, the Pareto Set analysis by Level Diagrams can be used for the reduction of the Pareto Front by selecting solutions characterized by preferred values of selected decision variables. In this work, two Pareto Front and Set reduction methods have been proposed for application in succession; a multi-state system redundancy allocation case study of literature has shown their capabilities of leading to a significant reduction of the solutions to be considered by the decision maker.

Acknowledgments: The authors are thankful to Professor David Coit of Rutgers University for providing the Pareto Front and Set data. 


\subsection{REFERENCES}

[1] ATKOSoft (1997), Survey of Visualization Methods and Software Tools, http://europa.eu.int/en/comm/eurostat/research/supcom.96/30/result/a/visualisation_meth ods.pdf

[2] Blasco , X., Herrero, J.M., Sanchis, J., Martínez, M. (2008), A New Graphical Visualization of n-Dimensional Pareto Front for Decision-Making in Multiobjective Optimization, Information Science, an International Journal, Volume 178 , Issue 20: 3908-3924

[3] Busacca, P. G., Marseguerra, M. and Zio, E. (2001), Multiobjective Optimization by Genetic Algorithms: Application to Safety Systems, Reliability Engineering and System Safety, 72: 59-74.

[4] Kulturel-Konak, S., Smith, A. and Coit, D. W. (2003), Efficiently Solving the Redundancy Allocation Problem Using Tabu Search, IEEE Transactions, 35(6): 515-526.

[5] Levitin ,G. (2005), Universal generating function in reliability analysis and optimization, Springer-Verlag, London.

[6] Levitin, G. and Lisnianski, A. (2001), A New Approach to Solving Problems of MultiState System Reliability Optimization, Quality and Reliability Engineering International, 17(2):93-104.

[7] Lotov, A.V., Bushenkov, V.A. and Kamenev, G.K. (2004), Interactive Decision Maps, Approximation and Visualization of Pareto Frontier, Kluwer Academic Publishers.

[8] Messac, A, Wilson, B.H. (1998), Physical programming for computational control, AIAA Journal 36 (1): 219-226.

[9] Taboada, H. and Coit, D. (2007), Recent Developed Evolutionary Algorithms for the Multi-Objective Optimization of Design Allocation Problems, Proceedings of the $5^{\text {th }}$ International Conference on Quality \& Reliability (ICQR5), Chiang Mai, Thailand

[10] Taboada, H., Espiritu, J. and Coit, D. (2007), MOMS-GA: a Multiobjective Multi-State Genetic Algorithm for System Reliability Optimization Design Problems, IEEE Transactions on Reliability, 57(1):182-191

[11] Ushakov, I. (1986), A Universal Generating Function, Soviet Journal of Computer and System Sciences, 24(5):37-49. 